\title{
NK/T-cell lymphoma of bilateral adrenal glands in a patient with pyothorax
}

\author{
Tomohide Tsukahara ${ }^{1 *}$, Akira Takasawa' ${ }^{1}$, Masaki Murata' ${ }^{1}$, Kazuyoshi Okumura², Masato Nakayama², \\ Noriyuki Sato ${ }^{1}$ and Tadashi Hasegawa ${ }^{3}$
}

\begin{abstract}
Primary lymphoma of adrenal glands is rare, and non-B-cell lymphoma associated with pyothorax is also very rare. Here we report the first autopsy case of non-B-cell lymphoma in bilateral adrenal glands of a 79-year-old woman with pyothorax who had an aggressive clinical course. Immunohistochemically, tumor cells showed CD3+, CD45RO+, CD5-, CD7-, CD4-, CD8-, CD10-, CD20-, CD30-, CD79a-, CD138-, CD56-, granzyme B-, TIA-1+ and ALK-. In addition, tumor cells were strongly EBER1-positive by in situ hybridization. In genomic DNA of tumor cells, T-cell receptor rearrangements were not detected by southern blotting. We finally diagnosed this case as extranodal NK/T-cell lymphoma (nasal type).

Virtual Slides: The virtual slide(s) for this article can be found here: http://www.diagnosticpathology.diagnomx. eu/vs/8050621197741854.
\end{abstract}

Keywords: NK/T-cell lymphoma, Adrenal gland, EBV, Pyothorax, Autopsy

\section{Background}

Lymphoma in the adrenal gland is rare, accounting for less than $1 \%$ of non-Hodgkin lymphomas. The features of lymphoma in adrenal glands are: (i) peak age of onset is elderly (mean age of 68 years), (ii) bilateral adrenal glands are involved in $60 \%$ of cases, (iii) adrenal failure occurs in $66 \%$ of cases, (iv) B-cell type is predominantly observed in $90 \%$ of cases and (v) prognosis is poor [1]. On the other hand, pyothorax-associated lymphoma, which is also rare (only $2 \%$ of patients with pyothorax), commonly shows the following features: (i) pathogenesis is related to pyothorax resulting form tuberculosis and artificial pneumothorax, (ii) common site of the tumor is around the pleural cavity, (iii) period of onset is more than 20 years, (iv) B-cell type is typical and strongly positive for Epstein-Barr virus, and (v) prognosis is poor [2]. However, non-B-cell type lymphoma occurring in adrenal glands of a patient with pyothorax has not been reported.

Here we report the first case of NK/T-cell lymphoma in bilateral adrenal glands in a patient with pyothorax.

\footnotetext{
* Correspondence: tukahara@sapmed.ac.jp

${ }^{1}$ Department of Pathology, Sapporo Medical University, South-1, West-17, Chuo-ku, Sapporo 060-8556, Japan

Full list of author information is available at the end of the article
}

\section{Case presentation}

A 79-year-old Japanese woman presented with cough and bloody sputum in July 2011. She had been followed since 1990 by a pulmonologist under the diagnosis of chronic pyothorax resulting from tuberculosis, and warfarin had been administered under the diagnosis of atrial fibrillation since 2009. There was no obvious evidence of existing immunodeficiency.

At the initial presentation, a large cystic lesion, pleural fluid and bilateral independent adrenal tumors $(55 \mathrm{~mm}$ x $31 \mathrm{~mm}$ on the right side and $57 \mathrm{~mm}$ x $32 \mathrm{~mm}$ on the left side) were detected by thoracic and abdominal computed tomography (CT) scans, respectively (Figure 1A, 1B). Tuberculosis bacterium was not detected in the sputum using PCR. Serum LDH was elevated to 1,038 U/L. Although use of warfarin was immediately stopped and coagulation therapy was performed using carbazochrome sodium sulfonate hydrate and tranexamic acid, the symptoms were not improved. Nineteen days after initial presentation, bloody sputum was successfully decreased by bronchial artery embolization. The next day, however, hyponatremia (Na: $113 \mathrm{mEq} / \mathrm{L}$ ) occurred, followed by increases of serum IL-2R $(1,185 \mathrm{U} / \mathrm{mL})$ and serum NSE (117.9 ng/mL). An abdominal CT scan showed an increase in sizes of the bilateral adrenal 

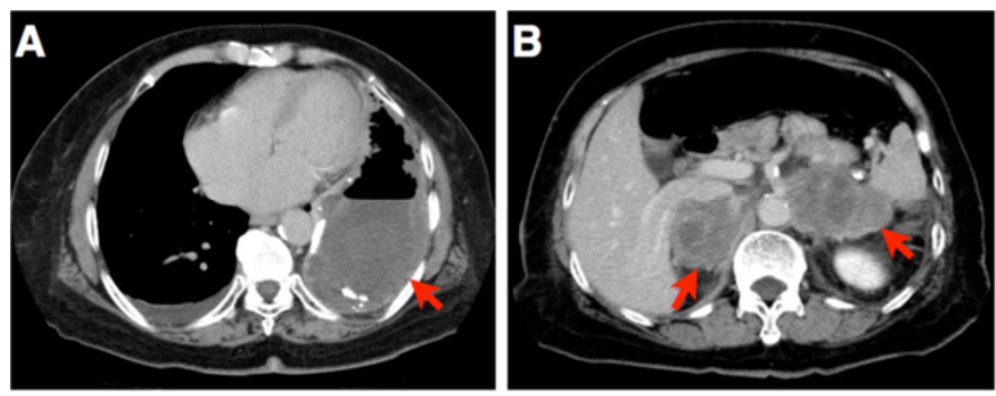

Figure 1 Enhanced computed tomography scan of the chest and abdomen. (A) Thoracic CT scan displayed large pyothorax and effusion in the left pleural cavity (indicated by a red arrow). (B) Abdominal CT scan displayed bilateral adrenal tumors (indicated by red arrows).

tumor masses $(57 \mathrm{~mm} \times 54 \mathrm{~mm}$ on the right side and $74 \mathrm{~mm} \times 45 \mathrm{~mm}$ on the left side). Two days later, under the diagnosis of adrenal failure caused by the tumors, augmentation of corticosteroid and correction of hyponatremia were started. Serum concentrations of cortisol, sodium and potassium were controlled well. However, seven days after the initiation of treatment, the patient had symptoms of general malaise and chest pain as well as atrial fibrillation with a rapid ventricular response. Serum LDH was increased to 3,650 U/L from 1,038 U/L at the initial presentation. Despite administration of antiarrhythmic agents, blood pressure decreased to less than $70 \mathrm{mmHg}$, and then cyanosis and hypouresis occurred. Thirty-three days after initial presentation (11 days after initiation of therapy for adrenal failure), the patient died. Autopsy was performed five hours after death.

\section{Materials and methods}

The autopsy specimen was fixed with $10 \%$ buffered formaldehyde and embedded in paraffin. Sections were cut to $2 \mathrm{um}$ in thickness and stained with hematoxylin and eosin. Immunohistochemistry was performed using primary antibodies against cytokeratin, vimentin, CD3, CD45RO, CD5, CD7, CD4, CD8, CD10, CD20, CD79a, CD138, CD56, granzyme B, TIA-1, ALK and Ki-67. Ki-67 index was calculated as the ratio of Ki-67-positive cell number to 1,000 tumor cells. In situ hybridization was performed using an anti-sense probe of Epstain-Barr virus (EBV)-encoded RNA 1 (EBER1). Southern blotting for detection of $\mathrm{T}$ cell receptor rearrangement in genome DNA of fresh frozen tumor tissue using probes against J $\mathrm{B} 1$ and $J ß 2$ of TCR $\beta$ chain and J $\gamma$ of TCR $\gamma$ chain was performed by BML, Inc (Tokyo, Japan).

\section{Results}

\section{Macroscopic findings}

In the left pleural cavity, pyothorax as a large cystic lesion (approximately $16 \mathrm{~cm} \times 7 \mathrm{~cm}$ ) containing necrotic tissues with pyogenic pleural fluid was detected. No tumoral lesion was detected around the left pleural cavity. In both adrenal glands, tumor masses $(90 \mathrm{~mm} \times 65 \mathrm{~mm}$ on the right and $85 \mathrm{~mm} \times 60 \mathrm{~mm}$ on the left) with necrotic change were detected (Figure 2). The right adrenal tumor had infiltrated into the right kidney and inferior vena cava, and the left adrenal tumor had infiltrated into the left kidney and spleen. Enlarged lymph nodes were not detected anywhere.

\section{Histologic and genomic findings}

The cystic wall of the pyothorax consisted of fibrous and hyalinizing tissue with a small population of infiltrating lymphocytes without atypia. No EBER1-positive cells were detected around the left pleural cavity by in situ hybridization. The adrenal tumors contained diffuse middle to large-sized neoplastic lymphoid cells with atypia and mitosis (Figure 3A). Both of the adrenal glands had completely disappeared due to invasion of the tumors. Immunohistochemically, the tumor cells were cytokeratin -, vimentin +, CD3+ (Figure 3B), CD45RO+, CD5-, CD7-, CD4-, CD8-, CD10-, CD20- (Figure 3C), CD30-, CD79a-, CD138-, CD56- (Figure 3D), granzyme B-, TIA-1 cytotoxic granule-associated RNA binding protein (TIA-1) + (Figure 3E) and ALK-. As shown in Figure 3F, Ki-67 was strongly positive in tumor cells (Ki-67 index: 77.0\%). Results of in situ hybridization showed that EBER1 was strongly positive in most of the tumor cells (Figure 3G). TCR rearrangements in genomic DNA of fresh frozen tumor cells were not detected in TCR $B$ and TCR $\gamma$ chains by southern blotting (Figure $3 \mathrm{H}$ ).

These findings indicated that the tumor had characteristics of peripheral T-cell origin or natural killer (NK)/T-cell origin. The findings of $\mathrm{CD} 3+, \mathrm{CD} 45 \mathrm{RO}+$ and CD56could indicate peripheral T-cell lymphoma. In contrast, the findings of TIA-1+, EBER1+ and no TCR rearrangement could indicate NK/T-cell lymphoma. Considering the findings of TIA-1+, EBER1+ and no TCR rearrangement, histological type of the tumor was finally diagnosed as CD56-negative extranodal NK/T-cell lymphoma (nasal type). 


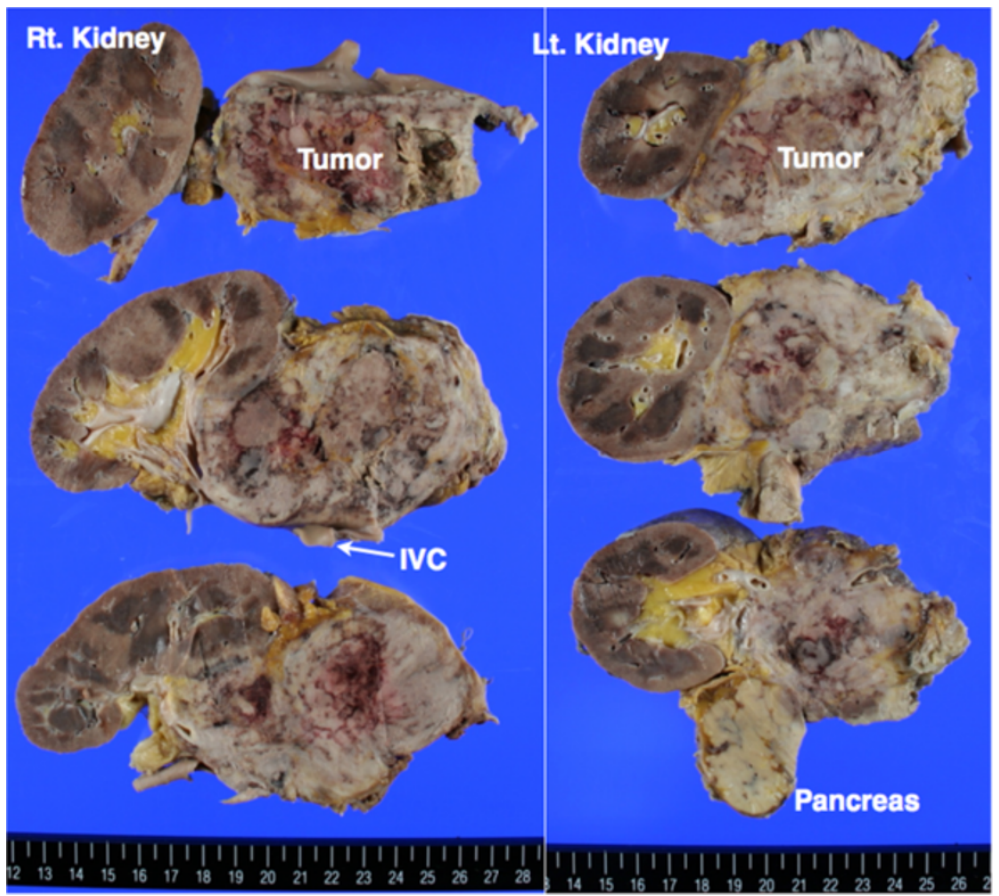

Figure 2 Macroscopic view of bilateral adrenal masses and adjacent organs.

\section{Discussion}

This is the first case of non-B-cell lymphoma in bilateral adrenal glands in a patient with pyothorax. Tumor cells had the characteristics of both peripheral T-cell lymphoma and NK/T-cell lymphoma. In addition, no tumor lesions were detected around the pyothorax. The tumor showed highly malignant characteristics and showed an aggressive clinical course.

Commonly, adrenal lymphoma shows histological characteristics of B-cell type, mainly diffuse large B-cell lymphoma (DLBCL). Therefore, this case of non-B-cell lymphoma is a rare case. Six cases of peripheral T-cell lymphoma and five cases of NK/T-cell lymphoma have been reported [3,4]. On the other hand, most pyothoraxassociated lymphomas have shown findings of DLBCL occurring in the pleural cavity in addition to strong positive findings of EBV. Nine cases of peripheral T-cell lymphoma associated with pyothorax have been reported [5-13], but no cases of NK/T-cell lymphoma associated with pyothorax have been reported. Only one case of lymphoma occurring in both adrenal glands in a patient with pyothorax has been reported [14]. In that case, a small lesion of lymphoma was also detected around the pyothorax lesion, and the histological type was diagnosed as DLBCL. Thus, non-B-cell lymphoma in adrenal glands in a patient with pyothorax such as the present case is an extremely rare condition.
For differential diagnosis, (i) "peripheral T-cell lymphoma, not otherwise specified", (ii) "extranodal NK/T-cell lymphoma (nasal type)" and (iii) chronic active EpsteinBarr virus infection (CAEBV) were considered. As described above, the tumor had characteristics of both peripheral T-cell and NK/T-cell lymphomas. The findings of $\mathrm{CD} 3+\mathrm{CD} 45 \mathrm{RO}+\mathrm{CD} 56$ - could indicate peripheral T-cell lymphoma. On the other hand, the findings of cytotoxicity-associated molecule TIA-1+, no TCR rearrangement and EBV + could indicate NK/T-cell lymphoma. The present case could not strictly be categorized into either. However, considering the findings of TIA-1+, EBER1+ and no TCR rearrangement, we finally diagnosed this lymphoma as extranodal NK/T-cell lymphoma (nasal type). Recently, Miles et al. reported a case of CD56negative extranodal NK/T-cell lymphoma [15]. In that case, neoplastic lymphoid cells expressed CD3, TIA-1 and EBER1 with an unusual lack of CD56. In addition, the patient had no typical symptoms of infectious mononucleosis-like illness, hypersensitivity to mosquito bites or other symptoms supporting CAEBV [16].

Our patient died only 33 days after initial presentation. Generally, the prognosis of both peripheral T-cell lymphoma and NK/T-cell lymphoma is poor. Nonnasal NK/Tcell lymphoma, such as this case, shows an aggressive clinical course. Chen et al. reported that the mean survival period of nonnasal NK/T-cell lymphoma patients was 


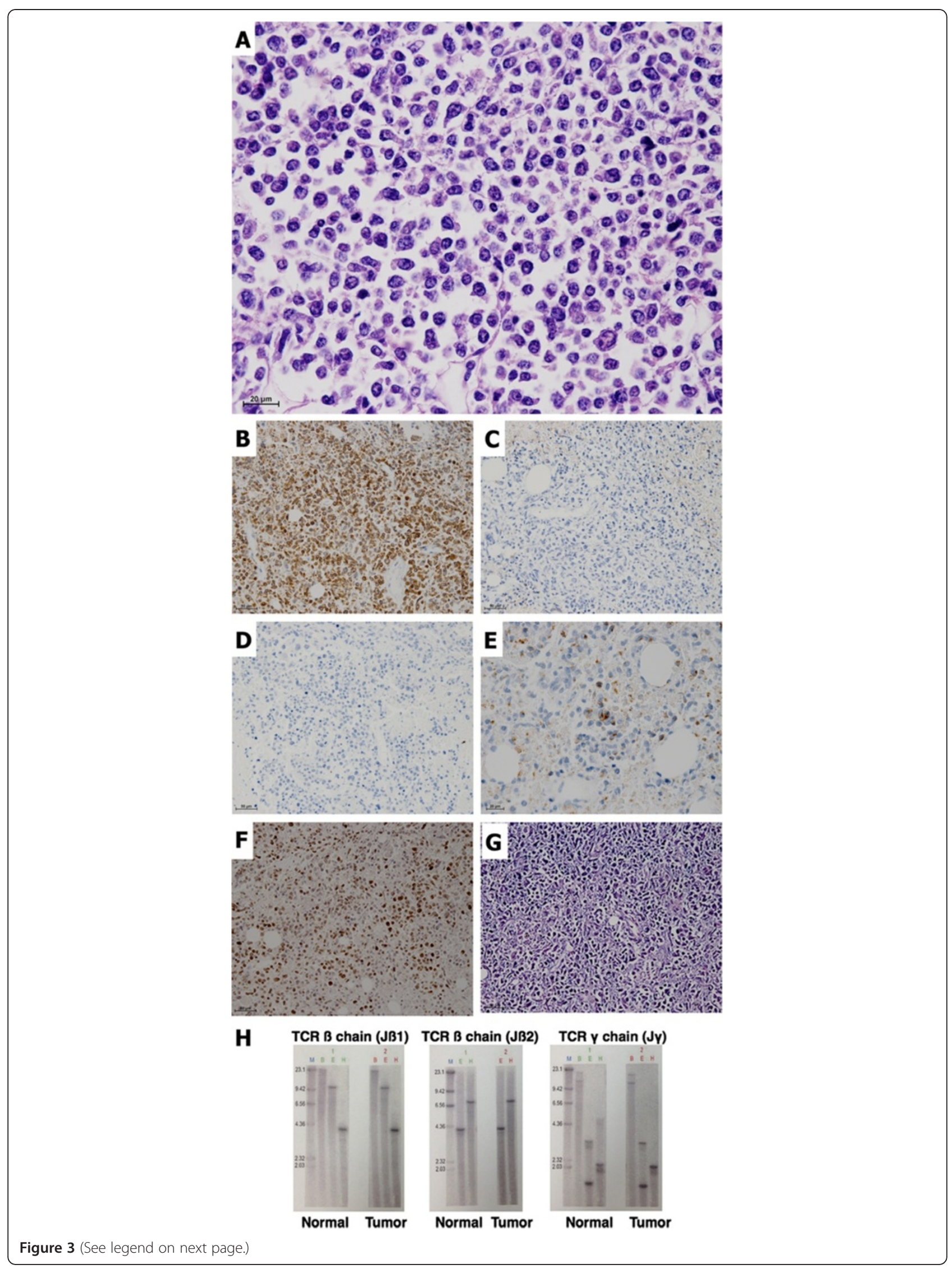


Figure 3 Microscopic views and T-cell receptor rearrangement of the tumors. HE (A) and immunohistochemistry against CD3 (B), CD20 (C), CD56 (D), TIA-1 (E) and Ki-67 (F). (G) In situ hybridization against EBER-1. (H) Southern blotting to detect T-cell receptor rearrangement in fresh frozen tumor tissue. The first column in each blot indicates the size of the DNA fragment (kbp). Restriction enzymes used were as the follows: $E_{\text {, }}$ EcoRl; B, BamHI and H, Hindlll.

3.5 months (from 1 week to 3 years) after initial presentation [17].

In pyothorax-associated lymphoma, transformation of lymphocytes caused by EBV infection and proliferative stimulation via inflammatory cytokines including interleukin6 in the microenvironment of chronic pyothorax might be the major cause of tumorigenesis [8]. In a narrow sense, the association between pyothorax and the present lymphoma of adrenal glands is still unknown because no tumor lesions were detected in the pleural cavity. Obviously, there is a possibility that the pyothorax existed incidentally. Nevertheless, the possibility that both the long-standing pyothorax lesion and EBV infection contributed to the tumor-initiating ability of tumor cells in the present case cannot be ruled out. Asakage et al. reported a case of EBV-positive T-cell lymphoma of the stomach in a patient with pyothorax. In that patient, no tumors were detected around the pleural cavity, as in the present case [5].

\section{Conclusion}

We have reported the first case of CD56-negative extranodal NK/T-cell lymphoma in bilateral adrenal glands in a patient with pyothorax. The tumor displayed highly malignant characteristics with a distressful clinical course. There is a possibility that chronic inflammation in the microenvironment of the pyothorax and EBV infection contributed to the tumorinitiating ability of tumor cells in the present case.

\section{Consent}

Written informed consent was obtained from the family of the patient for publication of this case report and any accompanying images. A copy of the written consent is available for review by the Editor-in-Chief of this journal.

\section{Competing interests}

The authors declare that they have no competing interests.

\section{Authors' contributions}

TT, AT and MM performed the autopsy and assessed macroscopic and microscopic findings. $\mathrm{KO}$ and $\mathrm{MN}$ treated the patient and contributed to acquisition of clinical data. TT and AT drafted the manuscript. NS and TH participated in the coordination. All authors read and approved the final manuscript.

\section{Author details}

'Department of Pathology, Sapporo Medical University, South-1, West-17, Chuo-ku, Sapporo 060-8556, Japan. ²Department of Surgery, Tenshi Hospital, North-12, East-3, Higashi-ku, Sapporo 065-0012, Japan. ${ }^{3}$ Division of Clinical Pathology, Sapporo Medical University Hospital, South-1, West-16, Chuo-ku, Sapporo 060-8543, Japan.

Received: 31 July 2012 Accepted: 26 August 2012

Published: 29 August 2012

\section{References}

1. Singh D, Kumar L, Sharma A, Vijayaraghavan M, Thulkar S, Tandon N: Adrenal Involvement in Non-Hodgkin's Lymphoma: Four Cases and Review of Literature. Leuk Lymphoma 2004, 45:789-794.

2. Aozasa K: Pyothrax-associated lymphoma. J Clin Exp Hematopathol 2006, 46:5-10.

3. Tsai W, Hsieh C, Cheng M, Lin C: Adrenal insufficiency in T-cell lymphoma. Int J Urol 2006, 13:794-797.

4. Kang S, Kim W, Lee K, Baek H, Park T, Jin H: Mainly adrenal gland involving NK/T-cell nasal type lymphoma diagnosed with delay due to mimicking adrenal hemorrhage. J Korean Med Sci 2011, 26:1386-1390.

5. Asakage N, Yamamoto T, Suzuki T, Haraguch Y, Tsukada K, Kobayashi S, Yamasaki S: A case of malignant T-cell lymphoma of gastric origin accompanied by pyothrax. Case Rep Gastroenterol 2009, 3:162-168.

6. Santini M, Fiorello A, Vicidomini G, Busiello L, Baldi A: A surgical case of pyothorax-associated lymphoma of T-cell origin arising from the chest wall in chronic empyema. Ann Thorac Surg 2009, 88:642-645.

7. Narimatsu H, Ota Y, Kami M, Takeuchi K, Suzuki R, Matsuo K, Matsumura T, Yuji K, Kishi Y, Hamaki T, Sawada U, Miyata S, Sasaki T, Tobinai K, Kawabata M, Atsuta Y, Tanaka Y, Ueda R, Nakamura S: Clinicopathological features of pyothorax-associated lymphoma; a retrospective survey involvinv 98 patients. Ann Oncol 2007, 2007:122-128.

8. Hashizume T, Aozasa K, Tomita Y, Matsushita K: Pyothorax-associated T-cell lymphoma: a case report. Jpn J Clin Oncol 2003, 33:145-147.

9. Kamiya I: A case of T-cell malignant lymphoma developing in the chest wall of chronic pyothorax after artificial pneumothorax. Nihon Kyobu Geka Gakkai Zasshi 1996, 44:709-713.

10. Nakamura S, Sasajima Y, Koshikawa T, Kitoh K, Kato M, Ueda R, Mori S, Suchi T: Ki-1 (CD30) positive anaplastic large cell lymphoma of T-cell phenotype developing in association with long-standing tuberculous pyothorax: report of a case with detection of Epstein-Barr virus genome in the tumor cells. Hum Pathol 1995, 26:1382-1385.

11. Fukino S, Fukata $T$, Inoue A, Hatazawa $Y$, Morio S: A surgical case of T-cell non-Hodgkin's lymphoma originating in the wall affected by chronic tuberculous empyema following artificial pneumothorax. Nihon Kyobu Geka Gakkai Zasshi 1992, 40:113-117.

12. Iuchi K, Aozasa K, Yamamoto S, Mori T, Tajima K, Minato K, Mukai K, Komatsu H, Tagaki T, Kobashi Y: Non-Hodgkin's lymphoma of the pleural cavity developing from long-standing pyothorax. Summary of clinical and pathological findings in thirty-seven cases. Jpn J Clin Oncol 1989, 1989:249-257.

13. Tsuruhara K, Sakaki T, Oyaizu N, Morii S: An autopsy case of an extranodal malignant lymphoma occurring primarily in the chest wall. Gan No Rinsho 1987, 33:1287-1293.

14. Nokubi M, Saito K, Kanai N, Fujii T, Nakagami H, Izumi T, Ohkawara K: An autopsy case of pyothorax-associated pleural lymphoma, initially presenting with bilateral adrenal mass. Byori to Rinsyo 1996, 1996: 529-533.

15. Miles R, Afify Z, Yaish H, Perkins S: CD56-negative extranodal nasal type NK/T-cell lymphoma. Pediatr Blood Cancer 2010, 55:186-189.

16. Kimura H: Pathogenesis of chronic active Epstein-Barr virus infection: is this an infectious disease, lymphoproliferative disorder, or immunodeficiency? Rev Med Virol 2006, 16:251-261.

17. Chan JK, Sin VC, Wong KF, Ng CS, Tsang WY, Chan CH, Cheung MM, Lau $\mathrm{WH}$ : Nonnasal lymphoma expressing the natural killer cell marker CD56: a clinicopathologic study of 49 cases of an uncommon aggressive neoplasm. Blood 1997, 89:4501-4513.

doi:10.1186/1746-1596-7-114

Cite this article as: Tsukahara et al:: NK/T-cell lymphoma of bilateral adrenal glands in a patient with pyothorax. Diagnostic Pathology 2012 $7: 114$. 